\title{
Non-intrusive Density Measurements Applied to Gravity Currents Interacting with an Obstacle
}

\author{
Maria Chiara DE FALCO ${ }^{\bowtie}$, Claudia ADDUCE, and Maria Rita MAGGI \\ Department of Engineering, University Roma Tre, Rome, Italy \\ $\triangle$ mariachiara.defalco@uniroma3.it
}

\begin{abstract}
The dynamics of lock-release gravity currents interacting with a triangular barrier are investigated experimentally by applying non-intrusive density measurements based on image analysis, to measure the instantaneous density fields. The relevant parameter varied is the ratio between the height of the obstacle and the initial water depth. Results suggest that the image analysis based on a calibration curve is a suitable technique for the study of the gravity currents dynamics, which is strongly affected by the presence of a bottom obstacle depending on the relative obstacle height.
\end{abstract}

Keywords: gravity currents, image analysis technique, bottom obstacle, density fields.

\section{INTRODUCTION}

Gravity currents are density-driven flows caused by a density difference, which can be due to a temperature or salinity gradient or the presence of suspended sediments. In the latter case, these flows are known as turbidity currents and represent a geohazard to seafloor structures and offshore pipelines. For this reason, in lakes or in reservoirs, a barrier could be designed to stop or deviate the flow away from the structures, which can be damaged by an interaction with the current (De Cesare et al. 2001). Obstacles with a triangular cross-section are a good approximation to represent submerged barriers (Tokyay and Constantinescu 2015) and an accurate comprehension of the implications of the interference can help to improve the engineering models, depending on the parametric conditions characterizing these flows.

In the present study, an image analysis technique is used to evaluate the instantaneous density fields of a gravity current interacting with a triangular obstacle. Several experiments are performed with and without the obstacle and the effect of the relative obstacle height on the dynamics of the dense current is studied. 


\section{EXPERIMENTAL PROCEDURE}

Lock-exchange gravity currents are produced in a Perspex tank $3 \mathrm{~m}$ long, $0.2 \mathrm{~m}$ wide, and $0.3 \mathrm{~m}$ deep (Fig. 1). A lock-release technique was applied to generate gravity currents, by dividing the tank in two different volumes with a removable gate, placed at a distance $x_{0}=0.4 \mathrm{~m}$ from the left wall. The left part of the tank was filled with a saline mixture at density $\rho_{1}=1010 \mathrm{~kg} / \mathrm{m}^{3}$ while the right part of the tank was filled with fresh tap water at a measured density $\rho_{0}=1000 \mathrm{~kg} / \mathrm{m}^{3}$, where $\rho_{1}>\rho_{0}$ with a constant $\Delta \rho=\rho_{1}-\rho_{0}=10 \mathrm{~kg} / \mathrm{m}^{3}$. Experiments were performed first on the horizontal bed and then with a triangular obstacle placed at a distance $L_{0}$ from the gate. The main parameter varied is the relative obstacle height $R_{0}=h_{c} / d$ where $d$ is the obstacle height and $h_{c}=H / 2$ is the current height (Benjamin 1968).

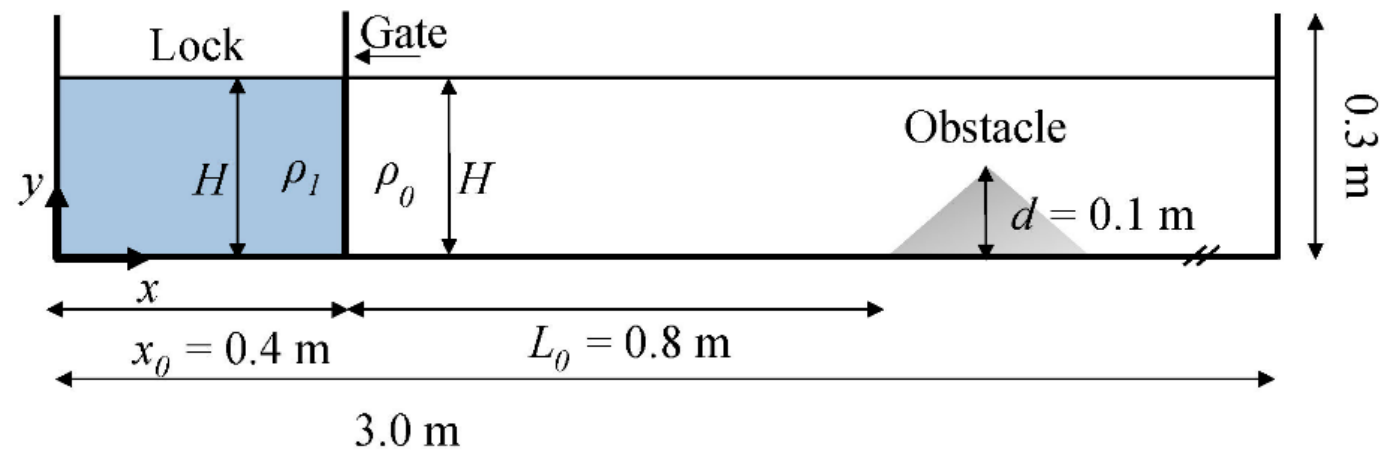

Fig. 1. Schematic representation of the experimental apparatus.

An image analysis technique is applied to evaluate the instantaneous density fields $\rho(x, y, t)$. The concentration of dye in the gravity current is considered linearly correlated to the salt concentration. A calibration technique is used to correlate the light intensity with the concentration of dye for each pixel of the acquired images. To this aim, nine controlled concentrations of dye were added and mixed to the fresh water in the tank in order to obtain a homogeneous dyed fluid and images were acquired to build a calibration curve. Figure 2 a shows the calibration points obtained for a single pixel in the domain, considering nine images.

A snapshot of a gravity current with $R_{\theta}=1.28$ and the corresponding instantaneous nondimensional density field $\rho^{*}(x, y)=\left(\rho(x, y)-\rho_{0}\right) / \Delta \rho$ are shown in Fig. 2b,c. The obtained density field gives insight into the gravity current's dynamics, which is strongly affected by the presence of the obstacle. A diluted current propagates downstream the obstacle, while part, denser, is retained by the obstacle.
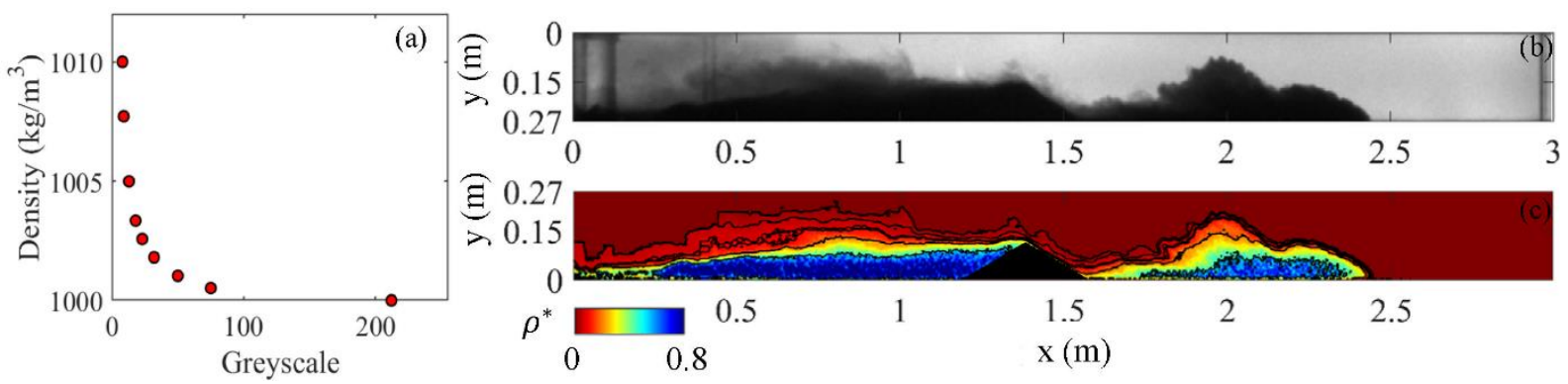

Fig. 2: (a) Calibration curve based on nine controlled quantities of dye, (b) snapshot of a gravity currents, and (c) corresponding non-dimensional density field after the interaction with the obstacle. 


\section{References}

Benjamin, T.B. (1968), Gravity currents and related phenomena, J. Fluid Mech. 31, 2, 209-248.

De Cesare, G., A. Schleiss, and F. Hermann (2001), Impact of turbidity currents on reservoir sedimentation, J. Hydraul. Eng. 127, 1, 6-16, DOI: 10.1061/(ASCE)0733-9429(2001)127:1(6).

Tokyay, T., and G. Constantinescu (2015), The effects of a submerged non-erodible triangular obstacle on bottom propagating gravity currents, Phys. Fluids 27, 5, 056601, DOI: 10.1063/1.4919384.

Received 22 March 2021

Accepted 21 April 2021 